\section{Typical and atypical antipsychotics increase risk of sudden cardiac death}

\section{QUESTION}

Question: What is the risk of sudden cardiac death with typical and atypical antipsychotics?

People: 279907 Medicaid enrolees, aged 34-74 years (mean 45.7 years; $65.2 \%$ women), who were either currently taking a single antipsychotic $(\mathrm{n}=93307)$ or no antipsychotics $(n=186600)$. Eligible antipsychotic users were those having at least one qualifying day of use of antipsychotic drugs during the study period. Two controls were randomly selected for each antipsychotic user, with matching for age, gender and first day of follow-up (defined as the first qualifying day for each antipsychotic user). Controls could subsequently become users of antipsychotic drugs. As well as the overall cohort, a subcohort matched for propensity scores was used in secondary analyses. Follow-up ended at the end of the study period, death, termination of Medicaid enrolment or if the participant subsequently met any of the study exclusion criteria. People could re-enter the study if they left it. Exclusion criteria: registration with Medicaid for $<2$ years; not eligible for full pharmacy benefits; not a regular user of medical care ( $<1$ filled prescription and $<1$ outpatient visit in each of the preceding 2 years); high risk of death from non-cardiac causes. Follow-up did not include time spent in hospital or the 30 days after discharge.

Setting: Tennessee, USA; 1 January 1990 to 31 December 2005.

Risk factors: Atypical or typical antipsychotic use. Current use of antipsychotic was defined as the days between filling the prescription until the prescribed medication would have run out, when the person was most likely to be taking the drugs. Indeterminate use was the period up to 90 days after finishing the current drug. Former use was defined as any time after the first antipsychotic use that was not classified as current or indeterminate use. Non-use was defined as any days without antipsychotic prescription and with no antipsychotic use in the past. Antipsychotic use was categorised by approximate chlorpromazine dose equivalents, with $<100 \mathrm{mg}$ chlorpromazine defined as low dose, 100-299 mg as moderate dose and $\geqslant 300 \mathrm{mg}$ as high dose. Typical antipsychotics assessed were thioridazine and haloperidol, and atypicals were clozapine, quetiapine, olanzapine and risperidone. Results were adjusted for demographic characteristics and comorbid conditions at baseline. Summary cardiovascular risk scores were calculated from baseline cardiovascular and somatic variables (score range 0-19, higher score indicating greater risk).

Outcomes: Sudden cardiac death occurring in the community, identified using death certificates.

\section{METHODS}

Design: Retrospective cohort study.

Follow-up period: 16 years.

\section{MAIN RESULTS}

There were 1870 sudden cardiac deaths during the study period, equivalent to 17.9 per 10000 person years. Current users of typical and atypical antipsychotics were significantly more likely to have sudden cardiac death than antipsychotic non-users (typical antipsychotics: incidence rate ratio (IRR) $1.99,95 \%$ confidence interval (CI) 1.68 to 2.34; atypical antipsychotics: IRR $2.26,95 \%$ CI 1.88 to 2.72 ). There was no significant difference between atypical and typical antipsychotics in risk of sudden cardiac death (IRR 1.14, 95\% CI 0.93 to 1.39). Former users of antipsychotics and non-users did not differ significantly in risk of sudden cardiac death (IRR 1.13, $95 \%$ CI 0.98 to 1.30). Current users of antipsychotics had a higher risk of sudden cardiac death than former users $(p<0.001)$. There was a dose-response relationship, with higher doses of typical or atypical antipsychotics increasing the risk of sudden cardiac death compared with non-users $(p<0.001$ for typical, $p=0.01$ for atypical antipsychotics). Similar results were found if the propensity matched subcohort was used.

\section{CONCLUSIONS}

Compared with antipsychotic non-users, current users of typical or atypical antipsychotics have a similarly increased risk of sudden cardiac death.

\section{ABSTRACTED FROM}

Ray WA, Chung CP, Murray KT, et al. Atypical antipsychotic drugs and the risk of sudden cardiac death. $N$ Engl J Med 2009;360:225-35.

Correspondence to: Dr Wayne A Ray, Department of Preventative Medicine, Village at Vanderbilt, Suite 2600, 1501 21st Avenue South, Nashville, TN 37212, USA; cindy.naron@vanderbilt.edu

Source of funding: National Heart, Lung, and Blood Institute and the Agency for Healthcare Quality and Research. 